\title{
NO HOMOGENEOUS TREE-LIKE CONTINUUM CONTAINS AN ARC
}

\author{
CHARLES L. HAGOPIAN ${ }^{1}$
}

\begin{abstract}
In 1960 R. H. Bing [4, p. 210] asked the following question. "Is there a homogeneous tree-like continuum that contains an arc?" We answer this question in the negative. This result generalizes Bing's theorem [4, p. 229] that every atriodic homogeneous tree-like continuum is arcless.
\end{abstract}

1. Introduction. Is every homogeneous tree-like continuum a pseudo-arc? This classic unsolved problem is one of the principal obstacles to the classification of homogeneous 1-dimensional continua [8].

Suppose there exists a homogeneous tree-like continuum $M$ that is not a psuedo-arc. F. B. Jones [14] proved that $M$ is indecomposable. Bing [3] proved that $M$ is not arc-like. C. E. Burgess [6] proved that $M$ is not circle-like. It follows from results of Burgess [7] and I. W. Lewis [15] that $M$ is not $k$-junctioned and that $M$ has a proper subcontinuum that is not a pseudo-arc. In this paper we prove that $M$ does not contain an arc.

2. Definitions and related results. A space is homogeneous if for each pair $x, y$ of its points there is a homeomorphism of the space onto itself that takes $x$ to $y$.

A chain is a finite collection $\left\{L_{i}: 1 \leqslant i \leqslant n\right\}$ of open sets such that $L_{i} \cap L_{j} \neq \varnothing$ if and only if $|i-j| \leqslant 1$. If $n>2$ and $L_{1}$ also intersects $L_{n}$, the collection is called a circular chain. Each $L_{i}$ is called a link.

A collection $\mathcal{R}$ of sets is coherent if for each nonempty proper subcollection $S$ of $\mathcal{R}$, an element of $\mathcal{S}$ intersects an element of $\mathcal{R} \backslash \mathcal{S}$.

Following Bing [2, p. 653], we define a finite coherent collection $\mathcal{T}$ of open sets to be a tree chain if no three elements of $\mathcal{T}$ have a point in common and no subcollection of $\mathcal{T}$ is a circular chain.

A continuum is a nondegenerate compact connected metric space.

A continuum $M$ is arc-like (circle-like, tree-like, respectively) if for each positive number $\varepsilon$ there is a chain (circular chain, tree chain) covering $M$ such that each element has diameter less than $\varepsilon$.

A continuum is decomposable if it is the union of two of its proper subcontinua; otherwise, it is indecomposable. If a continuum does not have a decomposable

Received by the editors October 19, 1982 and, in revised form, December 3, 1982.

1980 Mathematics Subject Classification. Primary 54F15, 54F20; Secondary 54F50, 54F55.

Key words and phrases. Homogeneity, tree-like continuum, arcless continuum, tree chain, hereditarily indecomposable continuum, pseudo-arc, dog-chases-rabbit principle.

' The author was partially supported by NSF Grant MCS 8205282 . 
subcontinuum it is hereditarily indecomposable. A continuum $M$ is a triod if $M$ has a subcontinuum $H$ such that $M \backslash H$ is the union of three nonempty disjoint open sets. If a continuum does not contain a triod it is atriodic. Since the plane does not contain uncountably many disjoint triods [17], every indecomposable homogeneous plane continuum is atriodic [10, Lemma 1].

Every homogeneous tree-like plane continuum is hereditarily indecomposable [11]. In fact, no homogeneous tree-like atriodic continuum has a decomposable subcontinuum [13, Corollary 2]. Furthermore, every homogeneous hereditarily indecomposable continuum is tree-like [18]. It would be interesting to know if every homogeneous tree-like continuum is hereditarily indecomposable [4, p. 210].

3. Preliminaries. Henceforth $M$ is a homogeneous continuum with metric $\rho$.

Let $\varepsilon$ be a positive number. A homeomorphism $h$ of $M$ onto $M$ is called an $\varepsilon$-homeomorphism if $\rho(x, h(x))<\varepsilon$ for each point $x$ of $M$.

Notation. Let $x$ be a point of $M$. We denote the set $\{y \in M$ : an $\varepsilon$-homeomorphism of $M$ onto $M$ takes $x$ to $y$ \} by $W(x, \varepsilon)$.

Lemma. For every positive number $\varepsilon$ and every point $x$ of $M$, the set $W(x, \varepsilon)$ is open in $M$.

Proof. This lemma follows from a short argument [10, Lemma 4, proof] involving E. G. Effros' topological transformation group theorem [9, Theorem 2.1].

Notation. Let $x$ be a point of $M$. Let $r$ and $s$ be numbers such that $0<r<s$. We denote the set $\{y \in M: r \leqslant \rho(x, y) \leqslant s\}$ by $A(x, r, s)$. The set $\{y \in M: \rho(x, y) \leqslant r\}$ is denoted by $B(x, r)$. We denote $\{y \in M: \rho(x, y)=r\}$ by $C(x, r)$.

4. Our result. In [4, p. 221] Bing proved that every homogeneous tree-like continuum has a proper subcontinuum that is not an arc. He then used this theorem to prove [4, p. 229] that no homogeneous tree-like atriodic continuum contains an arc. He also asked, "Is there a homogeneous tree-like continuum that contains an arc?” [4, p. 210]. We use the dog-chases-rabbit principle [5, p. 123] to answer Bing's question in the negative.

THEOREM. If $M$ is a homogeneous tree-like continuum, then $M$ does not contain an arc.

Proof. Assume $M$ contains an arc. Let $r_{1}, r_{2}, \ldots$ be a strictly decreasing sequence of numbers that converges to 0 . For each positive integer $i$, let $X_{i}=\{x \in M: x$ belongs to an arc in $M$ that has both endpoints in $\left.C\left(x, r_{i}\right)\right\}$.

It follows from the homogeneity of $M$ that

$$
M=\bigcup\left\{X_{i}: i=1,2, \ldots\right\} \text {. }
$$

For each $i$,

$$
X_{i} \text { is in the interior of } X_{i+1} \text { relative to } M \text {. }
$$

To see this let $x$ be a point of $X_{i}$, let $\varepsilon=\left(r_{i}-r_{i+1}\right) / 2$, and note that the open set $W(x, \varepsilon)$ of our Lemma is in $X_{i+1}$. 
It follows from (1), (2), and the compactness of $M$ that $X_{j}=M$ for some positive integer $j$. Assume without loss of generality that

$$
r_{j}>10 \text {. }
$$

For each $i$, let $Y_{i}$ be the set of all points $x$ of $M$ with the following property. There exist arcs $J$ and $K$ in $B(x, 9+1 / i)$ from $x$ to $C(x, 9+1 / i)$ such that

$$
\rho(J \cap A(x, 2-1 / i, 9+1 / i), K \cap A(x, 2-1 / i, 9+1 / i))>1 / i .
$$

It follows from (3) that

$$
M=\bigcup\left\{Y_{i}: i=1,2, \ldots\right\}
$$

For each $i$,

$$
Y_{i} \text { is in the interior of } Y_{i+1} \text { relative to } M \text {. }
$$

To see this let $x$ be a point of $Y_{i}$. Let $\varepsilon=(1 / i-1 /(i+1)) / 2$. For each point $y$ of $B(x, \varepsilon)$,

$$
\rho(A(y, 2-1 /(i+1), 9+1 /(i+1)), M \backslash A(x, 2-1 / i, 9+1 / i)) \geqslant \varepsilon .
$$

It follows that the open set $W(x, \varepsilon)$ is in $Y_{i+1}$. Hence (5) is true.

It follows from (4), (5) and the compactness of $M$ that for some positive integer $k$,

$$
Y_{k}=M \text {. }
$$

Let $\mathcal{T}$ be a tree chain covering $M$ such that each element of $\mathcal{T}$ has diameter less than $1 / k$. Let $x_{0}$ be a point of $M$. By (3), $C\left(x_{0}, 9\right) \neq \varnothing$. Let $\bigcup_{0}$ be a chain in $T$ that goes from $x_{0}$ to a point $x_{1}$ of $C\left(x_{0}, 9\right)$.

By (6), there exist arcs $J$ and $K$ in $B\left(x_{1}, 9\right)$ from $x_{1}$ to $C\left(x_{1}, 9\right)$ such that

$$
\rho\left(J \cap A\left(x_{1}, 2,9\right), K \cap A\left(x_{1}, 2,9\right)\right)>1 / k .
$$

Note that

$$
\text { either } J \text { or } K \text { misses } A\left(x_{1}, 3,9\right) \cap \cup \bigcup_{0} \text {. }
$$

To see this assume the contrary. Let $q$ be a subchain of $e_{0}$ that goes from a point of $J \cap A\left(x_{1}, 3,9\right)$ to $x_{1}$. Let $\mathcal{K}$ be a subchain of $\bigodot_{0}$ that goes from a point of $K \cap A\left(x_{1}, 3,9\right)$ to $x_{1}$. Either $\mathcal{G}$ is a subchain of $\mathscr{K}$ or $\mathscr{K}$ is a subchain of $\mathscr{G}$. Assume without loss of generality that $\mathcal{G}$ is a subchain of $\mathcal{K}$. Since $\mathcal{T}$ is a tree chain, $K$ intersects each link of $\mathscr{K}$. Hence the first link of $\mathcal{G}$ intersects both $J \cap A\left(x_{1}, 2,9\right)$ and $K \cap A\left(x_{1}, 2,9\right)$, and this contradicts (7). Thus (8) is true.

Assume without loss of generality that

$$
J \cap A\left(x_{1}, 3,9\right) \cap \cup \bigodot_{0}=\varnothing .
$$

Let $x_{2}$ be the endpoint of $J$ that belongs to $C\left(x_{1}, 9\right)$. Let $\bigcup_{1}$ be the chain in $\mathcal{T}$ that goes from $x_{1}$ to $x_{2}$. Since $\mathcal{T}$ is a tree chain,

$$
\text { each link of } \bigodot_{1} \text { intersect } J \text {. }
$$

Let $\mathscr{D}_{1}$ be the collection of links of $\bigodot_{1}$ that intersect $A\left(x_{1}, 4,9\right)$.

The sets $\cup \mathscr{D}_{1}$ and $\cup \bigodot_{0}$ are disjoint; for otherwise, by (9) and (10), there exists a circular chain in $e_{0} \cup e_{1}$, and this contradicts the fact that $\mathcal{T}$ is a tree chain. Hence $\mathscr{Q}_{1} \backslash e_{0} \neq \varnothing$. 
We proceed inductively. Let $n$ be a positive integer. Assume that for each positive integer $m$ less than or equal to $n$, we have defined points $x_{m}$ and $x_{m+1}$ of $M$ and subcollections $C_{m}$ and $\mathscr{D}_{m}$ of $\mathcal{T}$ such that

$$
\begin{gathered}
x_{m+1} \in C\left(x_{m}, 9\right), \\
\bigodot_{m} \text { is a chain from } x_{m} \text { to } x_{m+1},
\end{gathered}
$$

$\mathscr{Q}_{m}$ is the collection of links of $\mathcal{C}_{m}$ that intersect $A\left(x_{m}, 4,9\right)$,

$$
\left(\bigcup \mathscr{D}_{m}\right) \cap\left(\bigcup \bigcup\left\{\mathcal{C}_{i}: 0 \leqslant i<m\right\}\right)=\varnothing \text {, and }
$$

$\mathscr{D}_{m} \backslash \bigcup\left\{e_{i}: 0 \leqslant i<m\right\} \neq \varnothing$.

By (6), there exist arcs $J^{\prime}$ and $K^{\prime}$ in $B\left(x_{n+1}, 9\right)$ from $x_{n+1}$ to $C\left(x_{n+1}, 9\right)$ such that

$$
\rho\left(J^{\prime} \cap A\left(x_{n+1}, 2,9\right), K^{\prime} \cap A\left(x_{n+1}, 2,9\right)\right)>1 / k .
$$

It follows from (16) and the argument for (8) that either $J^{\prime}$ or $K^{\prime}$ misses $A\left(x_{n+1}, 3,9\right) \cap \cup \bigcup_{n}$. Assume without loss of generality that

$$
J^{\prime} \cap A\left(x_{n+1}, 3,9\right) \cap \cup \bigodot_{n}=\varnothing .
$$

Let $x_{n+2}$ be the endpoint of $J^{\prime}$ that belongs to $C\left(x_{n+1}, 9\right)$. Let $\bigodot_{n+1}$ be the chain in $\mathcal{T}$ from $x_{n+1}$ to $x_{n+2}$. Since $\mathcal{T}$ is a tree chain,

$$
\text { each link of } \bigodot_{n+1} \text { intersects } J^{\prime} \text {. }
$$

Let $\mathcal{D}_{n+1}$ be the collection of links of $e_{n+1}$ that intersect $A\left(x_{n+1}, 4,9\right)$.

Since $\mathcal{T}$ is a tree chain, it follows from (17) and (18) that

$$
\left(\bigcup \mathscr{D}_{n+1}\right) \cap\left(\cup e_{n}\right)=\varnothing \text {. }
$$

Furthermore,

$$
\left(\bigcup \mathscr{D}_{n+1}\right) \cap\left(\bigcup \bigcup\left\{\bigodot_{i}: 0 \leqslant i<n\right\}\right)=\varnothing .
$$

To see this suppose an element $L$ of $\mathscr{D}_{n+1}$ intersects $\cup \cup\left\{\Theta_{i}: 0 \leqslant i<n\right\}$. Let $\mathcal{E}$ be a chain in $U\left\{\bigcup_{i}: 0 \leqslant i<n\right\}$ such that $L$ intersects one end link of $\delta$ and $x_{n}$ belongs to the other end link of $\mathcal{E}$. Let $\mathscr{F}$ be the subchain of $\bigodot_{n+1}$ with the property that $x_{n+1}$ belongs to one end link of $\mathscr{F}$ and the other end link of $\mathscr{F}$ is $L$.

By $\left(14_{n}\right)$, links of $\mathcal{E}$ intersect links of $\bigodot_{n}$ only in $B\left(x_{n}, 4\right)$. By (19), links of $\mathscr{F}$ intersect links of $\bigodot_{n}$ only in $B\left(x_{n+1}, 4\right)$. Therefore, since $B\left(x_{n}, 4\right)$ and $B\left(x_{n+1}, 4\right)$ are disjoint, there exists a circular chain in $\bigodot_{n} \cup \mathcal{E} \cup \mathcal{F}$. This contradicts the fact that $\mathcal{T}$ is a tree chain. Hence (20) is true.

By (19) and (20), $\mathscr{D}_{n+1} \backslash \cup\left\{\bigodot_{i}: 0 \leqslant i \leqslant n\right\} \neq \varnothing$. This completes the inductive step.

For each positive integer $m$, there exist points $x_{m}$ and $x_{m+1}$ of $M$ and subcollections $C_{m}$ and $\mathscr{D}_{m}$ of $\sigma$ that satisfy $\left(11_{m}\right)-\left(15_{m}\right)$. Consequently $\sigma$ is infinite, and this contradicts the fact that $\mathcal{T}$ is a tree chain. Therefore $M$ does not contain an arc.

Comments. This argument can be modified to prove that no homogeneous tree-like continuum contains two continua with only one point in common. However the question of whether every homogeneous tree-like continuum is hereditarily indecomposable remains open. 
A continuum is a solenoid if and only if it is homogeneous and all of its proper subcontinua are arcs [12]. No tree-like continuum contains a solenoid. Perhaps every homogeneous continuum that contains an arc contains a solenoid.

ACKNOWLedgment. The author wishes to thank Mark Marsh, Jim Rogers, and Eldon Vought for several interesting conversations about homogeneous continua.

ADDENDUM. Recently Lewis [16] proved that if there is a homogeneous hereditarily indecomposable tree-like continuum that is not a pseudo-arc, then there is one with no arc-like subcontinua.

\section{REFERENCES}

1. R. H. Bing, A homogeneous indecomposable plane continuum, Duke Math. J. 15 (1948), 729-742.

2. __ Snake-like continua, Duke Math. J. 18 (1951), 653-663.

3. Each homogeneous nondegenerate chainable continuum is a pseudo-arc, Proc. Amer. Math. Soc. 10(1959), 345-346.

4. A simple closed curve is the only homogeneous bounded plane continuum that contains an arc, Canad. J. Math. 12 (1960), 209-230.

5: __ The elusive fixed point property, Amer. Math. Monthly 76 (1969), 119-132.

6. C. E. Burgess, Chainable continua and indecomposability, Pacific J. Math. 9 (1959), 653-659.

7. _ Homogeneous continua which are almost chainable, Canad. J. Math. 13 (1961), 519-528.

8. Homogeneous 1-dimensional continua, General Topology and Modern Analysis, Academic Press, New York, 1981, pp. 169-175.

9. E. G. Effros, Transformation groups and $C^{*}$-algebras, Ann. of Math. (2) 81 (1965), 38-55.

10. C. L. Hagopian, Homogeneous plane continua, Houston J. Math. 1 (1975), 35-41.

11. Indecomposable homogeneous plane continua are hereditarily indecomposable, Trans. Amer. Math. Soc. 224 (1976), 339-350.

12. A A characterization of solenoids, Pacific J. Math. 68 (1977), 425-435.

13. A A A A _riodic homogeneous continua, Pacific J. Math. (to appear).

14. , F. B. Jones, Certain homogeneous unicoherent indecomposable continua, Proc. Amer. Math. Soc. 2 (1951), 855-859.

15. I. W. Lewis, Almost chainable homogeneous continua are chainable, Houston J. Math. 7 (1981), 373-377.

16. The pseudo-arc of pseudo-arcs is unique, preprint.

17. R. L. Moore, Concerning triodic continua in the plane, Fund. Math. 13 (1929), 261-263.

18. J. T. Rogers, Jr., Homogeneous, hereditarily indecomposable continua are tree-like, preprint.

Department of Mathematics, California State University, SaCramento, California 95819 